$\$=$

\title{
Multi drug resistant (MDR) tuberculous meningitis with hydrocephalus treated with ventriculo-peritoneal shunting: a review
}

\author{
Vinod K. S. Gautam ${ }^{1 *}$, Sarbjeet Khurana ${ }^{2}$, Ravinder Singh ${ }^{3}$ \\ ${ }^{1}$ Assistant Professor, Department of Neurosurgery, Institute of Human Behaviour and Allied Sciences, \\ Government of NCT Delhi, Dilshad Garden, Delhi-110095,India \\ ${ }^{2}$ Associate Professor \& Head, Department of Epidemiology, Institute of Human Behaviour and Allied Sciences, \\ Government of NCT Delhi,Dilshad Garden, Delhi-110095,India \\ ${ }^{3}$ Associate Professor \& Head, Department of Medical Anthropology, Institute of Human Behaviour and Allied Sciences, \\ Government of NCT Delhi, Dilshad Garden, Delhi-110095,India \\ *Corresponding author E-mail: drvksg@gmail.com
}

\begin{abstract}
Introduction: Tubercular meningitis (TBM) is one of the severe manifestations of extra pulmonary tuberculosis. Prognosis of cases of multidrug resistance TBM is even worse. We present report of three cases of MDR tuberculous meningitis with hydrocephalus to highlight the challenges in the management of these patients.

Materials and Methods: The present study was done was done at IHBAS, a tertiary care neuropsychiatry hospital in Delhi, India. Three patients of MDR CNS TB treated at neurosurgery department were included in this study. Study design was a descriptive case series.

Results: All three cases were treated with second line ATT and ventrriculoperitoneal shunting. After a regular follow up with clinical and neuroimaging vigil and with laboratory testing, these patients showed improvement.

Conclusion: The decision to start second line ATT to treat resistant cases of CNS TB is often delayed and increases the morbidity and mortality. Prompt and precise decision is possible with vigilant clinical observation, lab tests and neuroimaging. Close follow-up is mandatory for detecting any adverse drug effect and therapeutic response in patients of MDR-CNS TB.
\end{abstract}

Keywords: CNS Tuberculosis; Multi drug resistance; Ventriculo-peritoneal shunt.

\section{Introduction}

Globally, MDR-TB accounts for 5\% of all tuberculosis patients, $3.5 \%$ of new cases and $20.5 \%$ of re-treatment cases (Murthy, 2012; WHO, 2014). Drug-resistant TB may be due to genetic mutation that makes a drug ineffective against the mutant bacilli. It may be due to primary infection with drug-resistant strains or may develop during the course of the treatment, mainly due to inappropriate treatment (Murthy, 2012). MDR tuberculous meningitis is appearing worldwide and is difficult to diagnose and treat. There is a need to review the MDR-TB in meningeal infections (Byrd and Davis, 2007).

We present report of three cases of MDR tuberculous meningitis with hydrocephalus to highlight the challenges in the management of these patients. We felt that the prompt intervention at different stages of this disease decreases the morbidity and mortality of MDR-tuberculous meningitis.

\section{Materials and methods}

The present study was done at IHBAS, a tertiary care neuropsychiatry hospital in Delhi, India. Three patients of MDR CNS TB operated by the first author were included in this case series and are described below. Case record files were reviewed for clinical and radiological analyses. All the patients were investigated systematically through various diagnostic tests. Anti-tubercular therapy and neurosurgical procedures were done as per the required intervention according to the neurosurgical diagnosis and of the patient and evidence-based practice. The patients were assessed in the follow up both radiologically and clinically for two years. Case 1: A 16-year-old female had presented with history of abnormal behavior, blurring of vision, headache and vomiting for 15 days. She was treated for pulmonary tuberculosis about two years ago and took ATT for only three months. About 10 months ago she was diagnosed as a case of tuberculous meningitis and five drug regimen of SHRZE along with Dexamethasone was given for three months duration. She did not improve and second line ATT was given, elsewhere. She developed features of raised intracranial pressure and MRI brain with contrast was advised, which revealed inflammatory granulomas in both frontal and temporal lobes, right sylvian fissure and in a right cerebellar hemisphere with leptomeningeal enhancement and hydrocephalus (Fig.1). Right sided ventriculoperitoneal shunt surgery was done under general anesthesia. Patient improved following surgery and second line antitubercular therapy was continued for 18 months.

Case-2: A 20-year-old female, admitted with history of weight loss, generalized weakness, giddiness off and on fever, headache and vomiting of about 45 days, one episode of seizure about 10 
days ago and complaint of double vision since one day. She was diagnosed as a case of pulmonary tuberculosis and pleural effusion about four months ago for which she received four first line anti-tubercular drugs, HRZE regimen, and pleural tapping was done. On examination, she had right sided sixth nerve paresis, nystagmus and left sided hemiparesis of grade $4 / 5$. She was put on five drugs ATT and Streptomycin (S) were added to the HRZE regimen. Antiepileptic (phenytoin), cerebral decongestants (dexamethasone, glycerol and acetazolamide) were also prescribed. She was diagnosed as a case of tuberculous meningitis and hydrocephalus. Right sided ventriculoperitoneal shunt was done under general anesthesia. Per operative tapping of the lateral ventricle revealed raised CSF pressure and CSR was grossly clear. CSF examination did not reveal acid-fast bacillus (AFB) and was negative for any growth on culture. Patient improved following surgery and was discharged with no neurological deficit. Few days later, she had seizures and deterioration of conscious level. Despite continued ATT and other cerebral decongestants patient did not improve and a possibility of shunt malfunction was considered. So, left ventriculoperitoneal shunt was done without disturbing the right VP shunt. However, there was no improvement in patient's neurological status. MRI of the brain revealed new granulomas and basal exudates and cerebral infarcts in MRI brain. So, firstline drug was stopped and second line ATT comprising of Kanamycin (K), Cycloserine, Mono amino salicylic acid (P), Moxifloxacin (M) and Ethionamide (ET) was started, and patient showed clinical improvement.

Case-3: A 22 year old female presented with history of off and on fever, headache for last 2 years, and blurring of vision, diplopia and facial asymmentry for last 6 months. Headache was insidious onset, holo-caranial and gradually progressive. On examination, she had right sided supranuclear facial nerve paresis. She was initially treated elsewhere for typhoid and chronic meningitis with antibiotics and ATT (HRZE). Neuroimaging revealed ventriculomegaly and right sided ventriculo-peritoneal shunting was done under general anesthesia. Per operatively ventricular tapping showed raised CSF pressure and CSF was grossly clear. CSF was sent for cytology and biochemical investigation, Gram stain, AFB stain and AFB culture. No microorganism was seen on direct microscopy of CSF but Ziehl Neelsen stain revealed AFB positive bacilli. CSF culture report was negative for any growth for pyogenic bacteria but was positive for Mycobacterium tuberculosis. Patient could not afford sensitivity testing for all the first line and second line ATT drugs. Post operatively injection Streptomycin was added to the first line ATT regimen. Patient showed initial improvement following surgery. Later, patient developed drowsiness and weakness of the limbs. MRI of the brain and spinal cord ( Fig-2 and 3) revealed increased basal exudates, with brainstem infarct, cerebral granulomas and spinal arachnoiditis. First line ATT was stopped and dexamethasone and second line ATT comprising of Kanamycin (K), Cycloserine, Mono amino salicylic acid (P), Moxifloxacin (M) and Ethionamide (Et) was started. Patient showed significant clinical improvement during follow up on second line ATT.

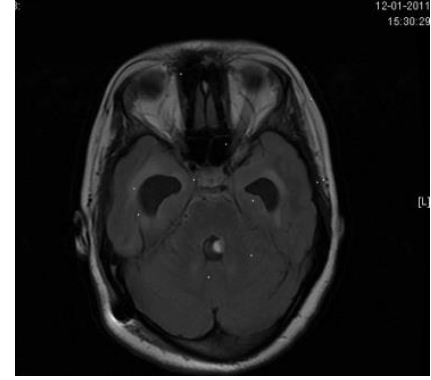

Fig. 1: MRI Brain FLAIR Axial View Image Showing Enlargement of Temporal Horns of Lateral Ventricle and Periventricular Edema.
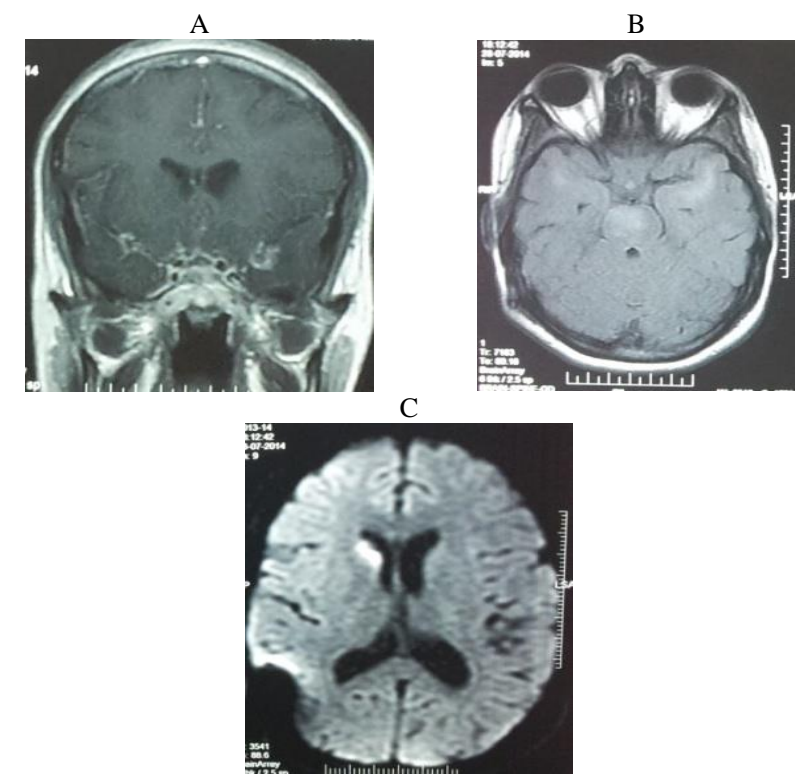

Fig. 2: MRI Brain: A. Coronal View Contrast Image Showing Basal Exudates, B. Axial View FLAIR Image of Brain Showing Hypointensity in Pons and Temporal Lobe, C. Diffusion Wighted Image Showing Infarct in Caudate Nucleus
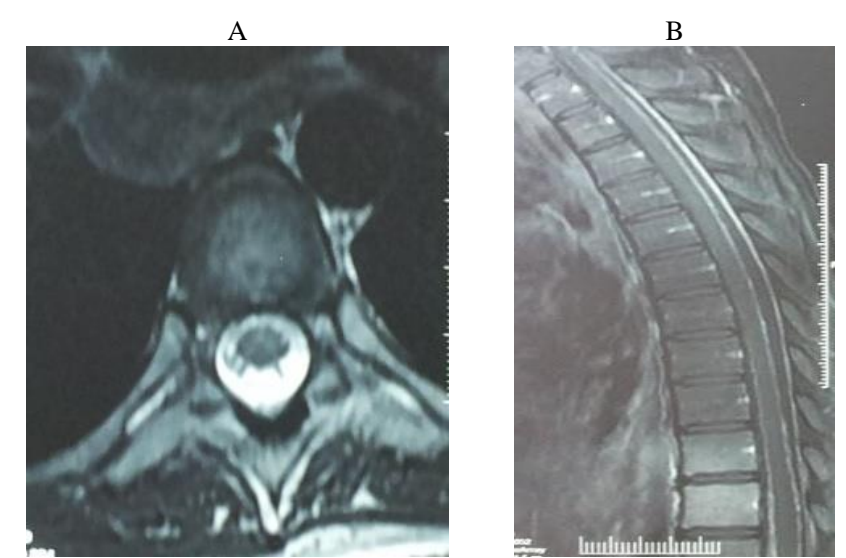

Fig. 3: T1 Contrast Image of the Dorsal Spine: A. Axial View and B. Sagittal View: Showing Leptomeningeal Enhancement and Increase in The CSF Subarachnoid Space.

\section{Discussion}

Clinical diagnosis of TBM is difficult as the clinical features are nonspecific and vary widely, and are often diagnosed when brain damage has already occurred (Murthy, 2010; Thwaites, 2005; Gautam, Khurana, Singh, 2013). Hydrocephalus is common sequelae of the tuberculous meningitis and is almost always present in patients who have had the disease for four to six weeks. Hydrocephalus is the commonest reason for neurosurgical referral in patients with TBM. It could be either of the communicating type or the obstructive type. Communicating type of hydrocephalus is more common complication in a patient of tubercular meningitis (Kamra, 2004). However, in our series all three patients had ob- 
structive hydrocephalus. Contrast enhanced CT scan or MRI of the brain is the imaging of choice as it provides adequate information regarding ventricular size, subependymal seepage, presence of infarcts and tuberculomas, and presence of basal exudates. The management of hydrocephalus can include medical therapy with dehydrating agents and steroids, and surgical treatment external ventricular drain, ventriculo-peritoneal shunt surgery or endoscopic third ventriculostomy (Gautam, Singh, Khurana, 2014).

$\mathrm{TB}$ of the central nervous system requires longer therapy preferably for one year. For any new case of CNS TB, it is advised that the initiation phase (IP) of two months should contain four drugs (HRZE) and continuation phase (CP) of 10 months should contain two drugs (HR). Continuation phase containing three drugs (HRE) as a daily regimen should be given only in countries with high levels of isoniazid resistance in new TB patients, and where isoniazid drug susceptibility testing in new patients is not done (or results are unavailable) before the continuation phase begins. Wherever feasible, the optimal dosing frequency is daily throughout the course of therapy. Daily dosing may help to prevent acquired drug resistance in TB patients starting treatment with isoniazid resistance (WHO, 2010). Adjuvant corticosteroid treatment (Dexammethasone or Prednisolone) should be given at least for one month in severe cases of CNS TB (Gautam, Khurana, Singh, 2013; NICE, 2007)). In tuberculous meningitis, ethambutol should be replaced by streptomycin. Neurosurgical intervention is reserved for management of late complications of CNS disease such as hydrocephalus or neurological involvement from Pott's disease (spinal TB). In our series, all three cases required VP shunt surgery as a CSF diversion procedure to treat obstructive hydrocephalus.

Patients whose prior course of therapy has failed have a high likelihood of MDR. Patients whose prior course of therapy has failed should therefore receive an empirical MDR regimen. New guidelines of WHO have emphasized the value of Drug susceptibility testing (DST) at the start of therapy for all previously treated patients with an aim of finding and treating multidrug-resistant TB (MDR-TB). The retreatment regimen with first-line drugs (formerly called "category 2" regimen) is ineffective in MDR-TB; it is therefore, critical to detect MDR-TB promptly so that effective regimen can be started.

Specimens for culture and drug susceptibility testing (DST) should be obtained from all previously treated TB patients at or before the start of treatment. DST should be performed for at least isoniazid and rifampicin (WHO, 2010). Drug susceptibility tests for TB are basically of two different types, phenotypical or genotypical. phenotypical meaning the observable characteristic of an organism, whereas genotypical is the genetic characteristics of the organism. Conventionally TB drug susceptibility has been phenotypic, involving the culturing of M. tuberculosis in the presence of TB drugs in order to detect growth (indicating drug resistance) or inhibition (indicating drug suscepbility ). The methods used are to perform direct or indirect tests on either solid or liquid media. Using conventional DST methods yields results within weeks (for liquid media using automated machine such as Bactec-320) or months (for solid media). Because of this delay, countries using conventional methods will need to start an empirical regimen while DST results are awaited. Since resistance arises from genetic mutations, another approach is to detect the mutations themselves. Many mutations associated with resistance have been identified and molecular methods to detect them have been developed. There are currently two main types of the genotypic based assay that have so far been endorsed by the WHO. These are Line-probe Hybridization Assays and molecular Beacon Assays. Line probe assays are tests that use PCR and reverse hybridization methods for rapid detection of mutations with rifampicin and isoniazid drug resistance Beacn assays detect $M$ tuberculosis complex and associated rifampicin resistance directly from the sample using ultra-sensitive PCR. The Xpert MTB/RIF assay is an automated real time based system (www.tbfacts.org). WHO also recommends Xpert TB test. Molecular tests can detect the resistance fast and line probe assay and X pert MTB/ RIF assay identifies rifampicin resistance, a marker of MDR (Lawn, 2011; Scott, 2011).). Ideally, the use of drugs in patients should be according to the drug susceptibility testing of the strain isolated from the patient as second-line drugs are less potent, more toxic and much more expensive. Initially at the time of presentation, clinically it is extremely difficult to suspect MDR-CNS TB, except where there is history of contact with MDR-TB patient (Murthy, 2012).

Since the drugs used for the treatment of MDR-TB are known to produce adverse effects, a proper pre- treatment evaluation and counseling are essential. The pre-treatment evaluation will include detailed history \& clinical examination, weight, height, complete blood count, including ESR, blood sugar to screen for diabetes mellitus; Liver Function Tests ( LFT), Blood Urea and S. Creatinine to assess the Kidney function ( KFT), TSH levels to assess the thyroid function, HIV counseling and testing, Urine examination - Routine and Microscopic, Pregnancy test (for all women in the child-bearing age group group) and chest X-Ray ( Posteroanterior view), USG abdomen, CT scan or MRI of the brain or spine.

Patients should be regularly screened for clinical status and adverse reactions, if any. For MDR treatment, anti-TB drugs are grouped according to efficacy, experience of use and drug class. All the first-line anti-TB drugs are in Group-1, except streptomycin, which is classified with the other injectable agents in Group 2. All the drugs in Groups 2-5 (except streptomycin) are second-line, or reserve, drugs.

Group-1 drugs are the most potent and best tolerated. If there is good laboratory evidence and clinical history that suggests that a drug from this group is effective, it should be used. All patients should receive a Group 2 injectable agent if susceptibility is documented or suspected. Among aminoglycoside, kanamycin or amikacin is the first choice of an injectable agent, given the high rates of streptomycin resistance in drug-resistant TB. In addition, both these agents are inexpensive, cause less ototoxicity than streptomycin, and have been used extensively for the treatment of drug- resistant TB.

Table 1: Detail of the Second Line ATT

\begin{tabular}{|c|c|c|}
\hline $\begin{array}{l}\text { Groups of } \\
\text { drugs }\end{array}$ & Types of Drugs & Name of Drugs \\
\hline Group 1 & First line oral agents & $\begin{array}{l}\text { Pyrazinamide(Z), } \\
\text { Ethambutol (E), } \\
\text { Rifabutin(Rfb) }\end{array}$ \\
\hline Group 2 & Injectable agents & $\begin{array}{l}\text { Kanamycin }(\mathrm{Km}), \\
\text { Amikacin }(\mathrm{Am}), \\
\text { Capreomycin }(\mathrm{Cm}), \\
\text { Streptomycin(S) }\end{array}$ \\
\hline Group 3 & Flouroquinolones & $\begin{array}{l}\text { Levofloxacin(Lfx), } \\
\text { Moxifloxacin(Mfx), } \\
\text { Ofloxacin(Ofx) }\end{array}$ \\
\hline Group 4 & $\begin{array}{l}\text { Oral bacteriostatic } \\
\text { second line agents }\end{array}$ & $\begin{array}{l}\text { Para-aminosalicylic acid(PAS), } \\
\text { Cycloserine(Cs), } \\
\text { Terizidone(Trd), } \\
\text { Ethionamide(Eto), } \\
\text { Protionamide(Pto) }\end{array}$ \\
\hline Group 5 & $\begin{array}{l}\text { Agents with unclear } \\
\text { role in treatment of } \\
\text { drug -resistant -TB }\end{array}$ & $\begin{array}{l}\text { Clofazimine, } \\
\text { linezolid(Lzd), Amoxicil- } \\
\text { lin/clavulanate(Amx/Clv), Thio- } \\
\text { actazone(Thz), } \\
\text { Imipenem/cilastatin(Ipm/Cln), } \\
\text { High -dose Isoniazid (High dose } \\
\text { H), } \\
\text { Clarithromycin(Clr) }\end{array}$ \\
\hline
\end{tabular}

(WHOReport:2010)

All patients should receive Group 3 medications if the $\mathrm{M}$. tuberculosis strain is susceptible or if the agent is thought to have efficacy. One of the higher generation fluoroquinolones, such as levofloxacin or moxifloxacin, is the fluoroquinolone of choice.

From Group, 4 Ethionamide (or protionamide) is often added to the treatment regimen because of its low cost. If cost is not a con- 
straint, p-aminosalicylic acid (PAS) may be added first. When two agents are needed, cycloserine can be added. Since the combination of ethionamide (or protionamide) and PAS of ten causes a high incidence of gastrointestinal side-effects and hypothyroidism, these agents are usually used together only when three Groups 4 agents are needed: ethionamide (or protionamide), cycloserine and PAS. Trazodone can be used instead of cycloserine and is assumed to be equally efficacious.

Group-5 includes agents with an unclear role in treatment of drug-resistant TB. Group-5 drugs are not recommended by WHOM for routine use in drug- resistant TB treatment because their contribution to the efficacy of multidrug regimens is unclear. They can be used in cases where it is impossible to design adequate regimens with the medicines from Groups $1-4$, such as in patients with XDR-TB.

Clinician must construct a regimen for each patient, and the individual patient-specific information should be part of the clinician's evaluation of each patient before starting the MDR regimen Treatment regimens should consist of at least four drugs with either certain, or almost certain, effectiveness. The clinical effectiveness or ineffectiveness of a drug cannot be predicted by DST with $100 \%$ certainty. Clinician should use any of the first-line oral agents from group1, an aminoglycoside or polypeptide by injection from group 2, a fluoroquinolone from group 3 and the remaining group four drugs to complete a regimen of at least four effective drugs. Four regimens with fewer than four effective drugs, consider adding two groups five drugs. The total number of drugs will depend on the degree of uncertainty, and regimens often contain five to seven.

The standardized regimen (STR) under Revised National Tuberculosis Control Program ( RNTCP) for pulmonary MDR-TB consists of an intensive phase of 6-9 months with six drugs, namely, Kanamycin, Ofloxacin, Ethionamide, Pyrizinamide, Ethambutol and Cycloserine, given daily. This is followed by continuation phase (CP) of 18 months of four drugs namely, Ofloxacin, Ethionamide, ethambutol and Cycloserine. At the end of six months, if culture remains positive, the intensive phase is extended further for three months (CTD, 2006). The intensive phase (IP) is defined by the duration of treatment with the injectable agent. The injectable agent should be continued for a minimum of six months, and for at least four months after the patient first becomes and remains smear- or culture-negative. Review of the patient's cultures, smears, X-rays and clinical status may also aid in deciding whether or not to continue an injectable agent longer than the above recommendation. Culture conversion (Conversion is defined as two consecutive negative smears and cultures taken 30 days apart.) After conversion, the minimum frequency recommended for bacteriological monitoring is monthly for smears and quarterly for cultures. Monitoring of MDR-TB patients by a clinician should be at least monthly until sputum conversion, then every 2-3 months also determines the overall duration of MDR treatment. These guidelines recommend continuing therapy for a minimum of 18 months after culture conversion. Extension of therapy to 24 months may be indicated in chronic cases with extensive pulmonary damage.

In view of lack of many studies, similar principles advocated by WHO for pulmonary disease may be applied for the management of MDR CNS-TB. In case of CNS-TB, monitoring is often difficult and clinicians have to rely on the clinical and radiological response to the therapy. It is unnecessary to continue first-line ATT after two months of IP if there is no clinical or radiological improvement. An effort should be made to get the pathological material like granulation tissue or plus for the culture and DST. Inability to get a pathological sample from a patient of CNS TB is a major problem. In the absence of demonstrable bacterial growth and definitive test results a thorough clinical evaluation and neuroimaging may guide course of the empirical second line MDR treatment, and the mainstay of management should be the clinical and neuroradiological response to the second-line ATT.

\section{Conclusion}

The clinical diagnosis of CNS TB is difficult due to nonspecific and varied clinical features. The access to the granulation tissue or tuberculoma is not possible in all the cases. So, the definitive or gold standard tests are not possible in most of the cases of CNS TB. As it is difficult to assess the therapeutic response and culture conversion in cases of in CNS-TB, the decision to start second line ATT to treat resistant cases is often delayed and increases the morbidity and mortality. The decision to stop first-line ATT and start the second line anti tubercular drugs should be prompt and precise. Vigilant clinical observation, lab tests and neuroimaging are the major decisive tools for the empirical therapy and detecting any adverse drug effect and therapeutic response during follow-up of the patients with MDR-CNS TB.

\section{References}

[1] Grange J M, Zumla A. Paradox of the global emergence of tuberculosis. Lancet. 1999; 353: 996. http://dx.doi.org/10.1016/S01406736(99)01196-4.

[2] Chandramukhi A, Lyashchenko K, Kumari HB, et al. Detection of antibodies to Mycobacterium tuberculosis protein antigen in cerebrospinal fluid of patients with tuberculous meningitis. J INF Dis. 2002; 186 (5):678-83.) http://dx.doi.org/10.1086/342293

[3] GB Migliori, R Loddenkemper, F Blasi and MC Raviglione. 125 years after Robert Koch's discovery of the tubercle bacillus: the new XDRTB threat. Is "science" enough to tackle the epidemic? Eur Respir J 2007; 29: 423-427. http://dx.doi.org/10.1183/09031936.00001307.

[4] Murthy J. Multi-drug-resistant central nervous system tuberculosis. Neurol India 2012; 60:143-145 http://dx.doi.org/10.4103/0028 3886.96381.

[5] WHO. Global tuberculosis report 2014. Geneva, Switzerland.

[6] Thomas F. Byrd, Larry E. Davis. Multidrug-resistant tuberculous meningitis. Current eurology and Neuroscience Reports. November 2007, Volume 7, Issue 6, pp 470-475.

[7] Murthy J. Tuberculous meningitis: The challenges. Neurol India 2010; 58:716-722 http://dx.doi.org/10.4103/0028-3886.72178.

[8] Thwaites, G. E., Bang N. D., Dung N. H., et al. 2005. The influence of HIV infection on clinical presentation, response to treatment, and outcome in adults with tuberculous meningitis. J. Infect. Dis.192:21342141. http://dx.doi.org/10.1086/498220.

[9] Gautam VKS, Khurana S., Singh R. Diagnostic and therapeutic challenges in the surgical management of CNS tuberculosis. Int J Med Health Sci. 2013, 2, 161-169.

[10]P. Kamra, R. Azad, K.N. Prasad, S. Jha, S. Pradhan, R.K. Gupta Infectious meningitis: prospective evaluation with magnetization transfer MRI. British Journal of Radiology, 77, 2004, 387-94. http://dx.doi.org/10.1259/bjr/23641059.

[11] Gautam VKS, Singh R, Khurana S. Reviewing Hydrocephalus: Personal experience of a neurosurgeon with ventriculoperitoneal shunts treatment. International Journal of Medicine, 2, 2014, 56-59. http://dx.doi.org/10.14419/ijm.v2i2.3219.

[12]NICE, 2006.http://www.nice.org.uk/guidance.

[13]WHO Report: Treatment of tuberculosis guidelines. 4th ed. 2010.

[14]WHO Report, 2006. www.who.int/maternal_child_adolescent.

[15]Lawn SD, Brooks SV, Kranzer K, Nicol MP, Whitelaw A, et al. Screening for HIV- associated tuberculosis and rifampicin resistance before antiretroviral therapy using the Xpert MTB/RIF assay: A prospective study. PLoS Med 2011;8: e1001067 http://dx.doi.org/10.1371/journal.pmed.1001067.

[16] Scott LE, McCarthy K, Gous N, Nduna M, Van Rie A, et al. Comparison of Xpert MTB/RIF with other nucleic acid technologies for diagnosing pulmonary tuberculosis in a high HIV prevalence setting: A prospective study. PLoS Med 2011;8: e1001061 http://dx.doi.org/10.1371/journal.pmed.1001061.

[17] Central TB Division (CTD), Directorate General of Health 2. Services, Ministry of Health and Family Welfare, Government of India. DOTSplus guidelines. New Delhi: CTD; 2006.

[18]http://www.tbfacts.org/drug-susceptibility.html. 